\title{
Effects of Pregnancy on Papillary Microcarcinomas of the Thyroid Re-Evaluated in the Entire Patient Series at Kuma Hospital
}

\author{
Yasuhiro Ito, Akira Miyauchi, Takumi Kudo, Hisashi Ota, Kana Yoshioka, Hitomi Oda, \\ Hisanori Sasai, Ayako Nakayama, Tomonori Yabuta, Hiroo Masuoka, Mitsuhiro Fukushima, \\ Takuya Higashiyama, Minoru Kihara, Kaoru Kobayashi, and Akihiro Miya
}

Background: An active-surveillance clinical trial of low-risk papillary microcarcinoma (PMC) patients has been performed at the authors' institution, Kuma Hospital, since 1993. Favorable oncological results have been reported. During the trial, a few patients were encountered with PMC that showed enlargement during pregnancy, and these cases have been reported. During pregnancy, a large amount of human chorionic gonadotropin (hCG) having weak thyrotropin (TSH) activity is produced, possibly affecting the progression of PMC. This study investigated how pregnancy and delivery influenced the progression of PMC in the entire active surveillance PMC patient series.

Methods: From 1993 to 2013, 1841 patients with low-risk PMC chose the active surveillance program. Fifty of the 1549 female PMC patients experienced 51 pregnancies/deliveries. To minimize observer variation, a single specialist sonographer re-evaluated the changes in the size of these 50 patients' PMCs before and after the pregnancies/deliveries.

Results: Four patients (8\%) showed enlargement of PMC by $\geq 3 \mathrm{~mm}$; one patient $(2 \%)$ showed a decrease by $\geq 3 \mathrm{~mm}$, and the remaining 44 patients (45 events, $90 \%$ ) showed stable disease. None of the patients had a novel appearance of lymph node metastases during pregnancy. Of the four patients with enlargement, two underwent surgery after delivery, and the other two continued the active surveillance, since their tumors did not grow after the delivery. After delivery, the PMC of one of these four patients remained stable, and another showed a decrease in PMC size. To date, six more PMC patients underwent surgery after delivery for reasons other than disease progression due to pregnancy and delivery: two opted out of active surveillance, two were identified with a nodal metastasis during active surveillance after delivery, one had Graves' disease, and one showed enlargement of nodules of the contralateral lobe.

Conclusions: Pregnancy and delivery was associated with an increase in size of PMCs in only $8 \%$ of the 51 pregnancies/delivery cases. None of the patients developed nodal metastasis during pregnancy. Thus, a possible future pregnancy does not prevent such patients from undergoing active surveillance, although watchful observation during pregnancy is recommended.

\section{Introduction}

A N ACTIVE SURVEILLANCE CLINICAL TRIAL of low-risk papillary microcarcinoma (PMC: i.e., a papillary carcinoma $\leq 1 \mathrm{~cm}$ ) was first proposed by Akira Miyauchi in 1993 and was initiated after the approval of other doctors at the authors' institution, Kuma Hospital, a 58-bed hospital in Kobe (population $>1.5$ million), Japan. This proposal was supported by both the considerably high incidence of small latent carcinomas detected in many autopsy studies (1) and the small thyroid carcinomas detected and diagnosed by ultrasound and fine-needle aspiration biopsy (FNAB) through screening in $3.5 \%$ of otherwise healthy women aged 30 years or older. This incidence was more than 1000 times higher than the prevalence of clinical thyroid cancer in Japanese women at that time (2).

(C) Ito et al. 2015; Published by Mary Ann Liebert, Inc. This Open Access article is distributed under the terms of the Creative Commons Attribution Noncommercial License (http://creativecommons.org/licenses/by-nc/4.0/) which permits any noncommercial use, distribution, and reproduction in any medium, provided the original author(s) and the source are credited. 
In 1995, the Cancer Institute Hospital in Tokyo, Japan, also initiated an active surveillance program, and the favorable results obtained at both hospitals were published between 2003 and 2014 (3-6). Studies in the United States and Korea revealed that the incidence of thyroid carcinomas (especially small-size papillary carcinomas) had greatly increased, although the mortality rate remained stable, indicating that many harmless small papillary carcinomas were detected and diagnosed in patients who then underwent unnecessary surgery (7-9).

Human chorionic gonadotropin (hCG) is produced especially in the early phase of pregnancy. Thyrotropin (TSH) and hCG share a common alpha subunit, and hCG has weak TSH activity (10-12). An important question is thus whether PMCs grow and become clinically important during pregnancy. If so, prophylactic surgery would be recommended for women with PMC who may become pregnant in the future.

Shindo et al. treated four cases of PMCs that apparently enlarged in size during the patients' pregnancies, and they reported that PMCs in pregnant women are likely to grow during pregnancy based on an analysis of a small number of cases in a limited period (13). Since this is a potentially important clinical issue, all PMC patients at Kuma Hospital who experienced pregnancy and delivery during the Hospital's active surveillance program were reviewed by checking the medical records as far as possible to exclude selection bias. The ultimate purpose of this study was not to investigate the changes in the size of PMCs during pregnancy, but rather to investigate whether women with low-risk PMCs and possible future pregnancies could be appropriate candidates for active surveillance, and this was done by comparing the size of PMCs before pregnancy with that after delivery. The possible appearance of nodal metastasis during pregnancy was also examined.

\section{Patients and Methods}

\section{Identifying the low-risk PMC patients who experienced pregnancy and delivery}

The definition of low-risk PMC excludes the following: the presence of distant metastasis or node metastasis on imaging studies, the presence or possibility of invasion to the trachea and/or the recurrent laryngeal nerve, and cytological findings of high-grade malignancy as described (6). Between 1993 and 2013, 1841 patients with low-risk PMCs were selected for active surveillance at Kuma Hospital. Of these, 1549 (84\%) were females, and 50 experienced pregnancy and delivery during their active surveillance. All tumors were cytologically diagnosed as papillary thyroid carcinomas.

Patients with missing data prior to pregnancy and/or after delivery, who had a miscarriage, or who underwent an abortion were excluded from the series. None of the patients with low-risk PMC underwent thyroid surgery during pregnancy. One patient experienced pregnancy and delivery twice. Thus, this study involved 50 patients and 51 pregnancy/delivery events in total. The patients' ages at delivery ranged from 28 to 41 years (median 33 years). Twenty-nine patients were positive for thyroid antibodies, and six of these patients had Graves' disease. Of the six patients with Graves' disease, three were administered propylthiouracil (PTU) during pregnancy, and the remaining three did not receive PTU due to disease remission at the time of pregnancy. Fifteen patients, including nine positive for antithyroid antibodies, were administered L-thyroxine to keep their TSH level at a low-normal value. The TSH level was measured once or more during pregnancy in 39 patients (40 events) and was maintained within the reference range (mostly lownormal) or mildly suppressed. Human use approval was obtained by the ethical committee, and informed consent was obtained from each patient.

\section{Ultrasound evaluation}

The PMCs had been evaluated by ultrasound one year or less before pregnancy in all patients to investigate size and the presence/absence of node metastasis. If multiple PMCs were observed, the size of the largest tumor was selected. All 50 of the patients were negative for node metastasis on ultrasound examination. Forty-eight patients (49 events) underwent an ultrasound evaluation within one year after delivery. The other two patients underwent an ultrasound evaluation at 22 and 26 months after delivery, respectively, because they did not visit the hospital until then. An ultrasound evaluation of the PMC was also performed during pregnancy in 32 patients (33 events) once or more.

As in previous studies, the size of a PMC was considered to have enlarged when the tumor size grew by $\geq 3 \mathrm{~mm}(5,6)$ compared with its size before pregnancy. For the present study, to minimize observer variation, the tumor size and the presence of node metastasis were re-evaluated on ultrasound images without the date information by a single sonographer (H.O.) who was both a specialist in ultrasound examination of the neck and the director of Kuma Hospital's ultrasound division.

\section{Results}

\section{Change in the size of PMC from before pregnancy to after delivery}

The changes in the size of the PMCs from before pregnancy to after delivery are summarized in Table 1 . The PMC enlarged by $\geq 3 \mathrm{~mm}$ in only $4 / 51$ cases ( $8 \%$ ), and the tumor decreased in one case (2\%) by $3 \mathrm{~mm}$. The remaining $46(90 \%)$ cases were classified as unchanged according to the criteria.

\section{Clinical courses of the four patients whose PMC enlarged during pregnancy}

Table 2 summarizes the profiles and clinical courses of the four patients showing size enlargement of their PMCs during

Table 1. Change in the Size of 50 PMC Cases from Before Pregnancy to After Delivery

\begin{tabular}{lllcccc}
\hline Size change & $\geq 3 \mathrm{~mm}$ & $+2 \mathrm{~mm}$ & $+1 \mathrm{~mm} \mathrm{to}-1 \mathrm{~mm}$ & $-2 \mathrm{~mm}$ & $-3 \mathrm{~mm}$ & Total \\
\hline No. of patients & $4(8 \%)$ & $1(2 \%)$ & $44(86 \%)$ & $1(2 \%)$ & $1(2 \%)$ & $51(100 \%)$ \\
\hline
\end{tabular}

PMC, papillary microcarcinoma. 
Table 2. Clinical Courses of the Four Patients Whose PMCs Enlarged by $\geq 3$ mm During Pregnancy

\begin{tabular}{ccccccc}
\hline Case & $\begin{array}{c}\text { Age at delivery } \\
\text { (years) }\end{array}$ & $\begin{array}{c}\text { Size at initial } \\
\text { diagnosis }(\mathrm{cm})\end{array}$ & $\begin{array}{c}\text { Size before } \\
\text { pregnancy }(\mathrm{cm})\end{array}$ & $\begin{array}{c}\text { Size after } \\
\text { delivery }(\mathrm{cm})\end{array}$ & Management & Histology \\
\hline 1 & 28 & 0.9 & 0.9 & 1.7 & Operation & Conventional \\
2 & 28 & 0.7 & 0.7 & 1.8 & Operation $^{\mathrm{b}}$ & Honeycomb-like $^{\mathrm{b}}$ \\
3 & 33 & 0.8 & 0.7 & 1.1 & Continuous surveillance $^{\mathrm{a}}$ & \\
4 & 35 & 0.7 & 0.7 & 1.0 & Continuous surveillance \\
\hline
\end{tabular}

${ }^{\mathrm{a}}$ The size decreased to $1.0 \mathrm{~cm}$ thereafter.

${ }^{\mathrm{b}}$ Papillary carcinoma with honeycomb-like multiple small cysts

pregnancy. Of these, two patients (cases 1 and 2) underwent surgery after their delivery because of the apparent size enlargement during pregnancy, while the other two patients have continued active surveillance to date (15 and 36 months after delivery) because their tumor sizes did not increase after delivery; the tumor actually decreased in one of these patients.

The PMC in case 1 was a solid tumor (Fig. 1), but the tumor of case 2 had multiple cystic lesions and displayed a special pathological type designated as papillary thyroid carcinoma with multiple honeycomb-like small cysts, as reported by Kobayashi et al. (14). The tumor had grown to $18 \mathrm{~mm}$ at one month after delivery, but the carcinoma was almost cystic

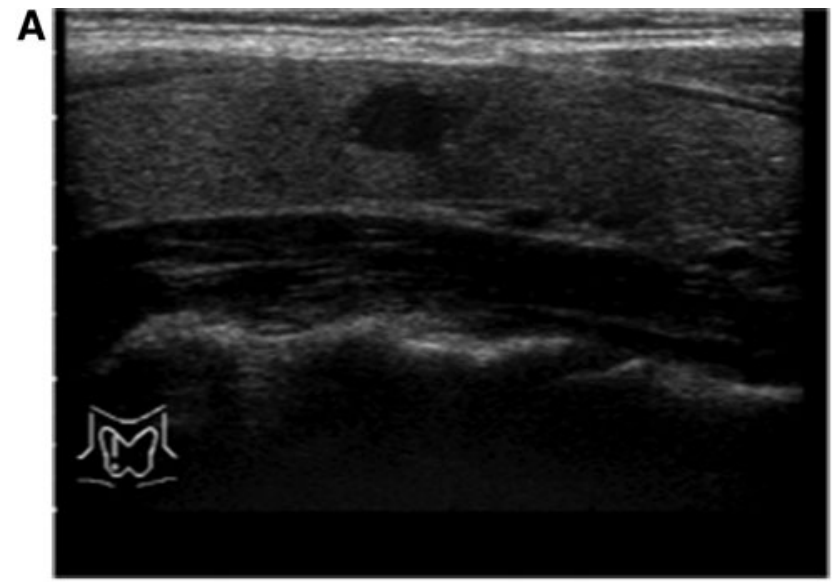

B

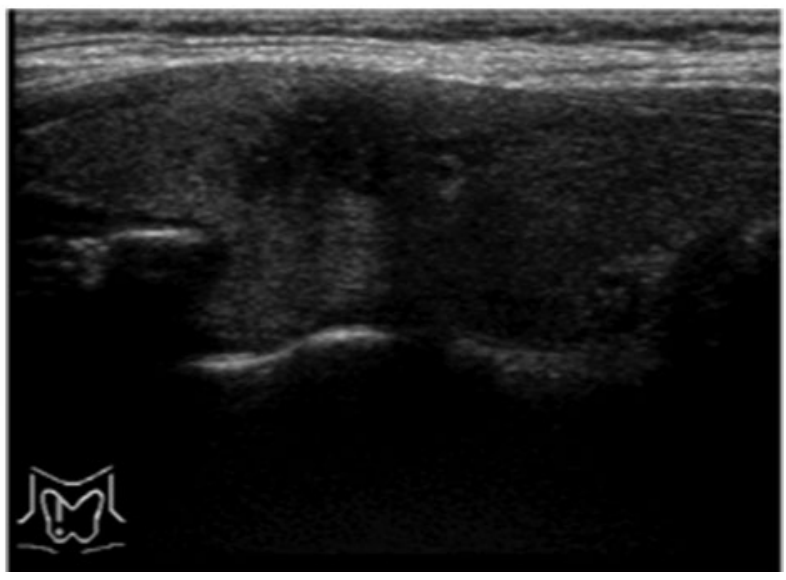

FIG. 1. Ultrasonographic findings of the papillary microcarcinoma (PMC) of patient 1 before pregnancy (A) in April 2009 and after delivery (B) in May 2010. The tumor was solid, and its histology was conventional papillary carcinoma.
(Fig. 2). The size decreased to $12 \mathrm{~mm}$ at two months after delivery (just before surgery) because of the shrinkage of the cystic components.

Case 1 showed an enlargement of the PMC size in the 1st and 2nd trimesters. The size of the PMC was not measured during the pregnancy in case 2 .

The PMC of case 3 enlarged by $3 \mathrm{~mm}$ in the 1 st trimester, diminished by $2 \mathrm{~mm}$ in the 3rd trimester, but enlarged again by $3 \mathrm{~mm}$ after delivery compared with its before-pregnancy

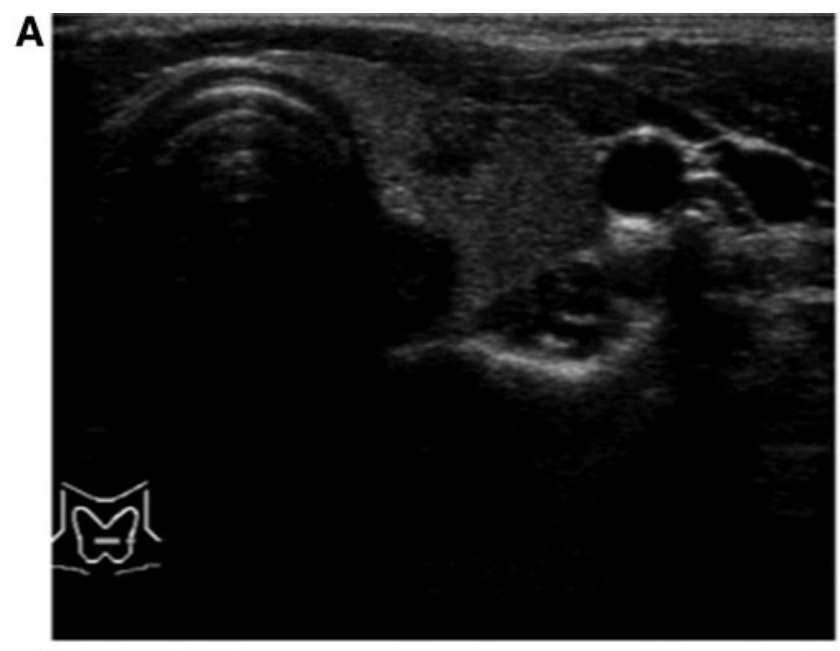

B

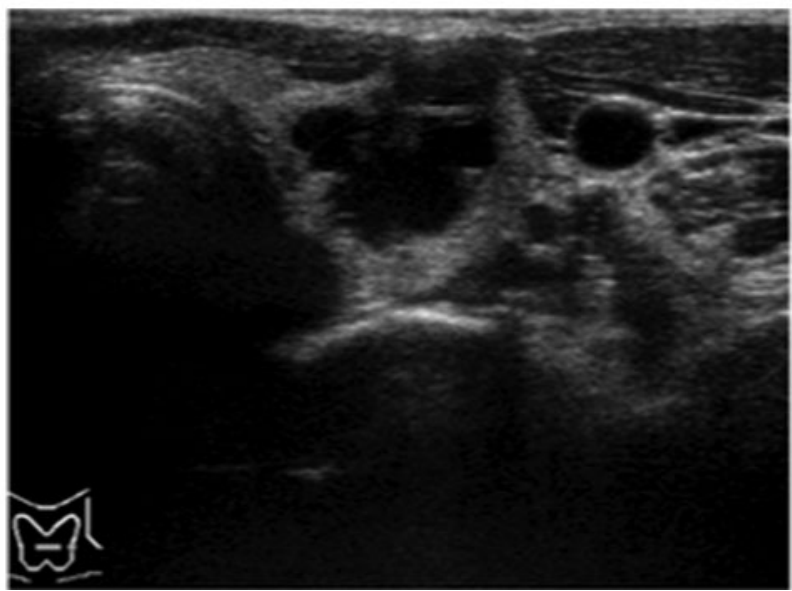

FIG. 2. Ultrasonographic findings of the PMC of patient 2 before pregnancy (A) in July 2009 and after delivery (B) in August 2010. The tumor was predominantly cystic, and the histology was papillary carcinoma with multiple honeycomblike small cysts. 
Table 3. Surgical and Pathological Profiles of the Eight PMC Patients who Underwent Surgery After Delivery

\begin{tabular}{lcclcrrrr}
\hline Case & $\begin{array}{c}\text { Age at surgery } \\
\text { (years) }\end{array}$ & Thyroidectomy & Node dissection & Ex and $p E x$ & $c N$ & $p N$ & Ki-67 LIs (\%) & Histology \\
\hline $1^{\mathrm{a}}$ & 28 & Total & Level VI & None & 0 & $1 \mathrm{a}$ & $<5$ & Conventional \\
$2^{\mathrm{a}}$ & 28 & LI & Level VI & None & 0 & 0 & $<5$ & Honeycomb-like \\
3 & 29 & LI & Level VI & None & 0 & 0 & $<5$ & Conventional \\
4 & 32 & Total & Level VI & None & 0 & 0 & $<5$ & Conventional \\
5 & 33 & Total & Level VI & None & $1 \mathrm{a}$ & $1 \mathrm{a}$ & $<5$ & Conventional \\
6 & 43 & Total & Level II-IV, VI & None & $1 \mathrm{~b}$ & $1 \mathrm{~b}$ & $<5$ & Conventional \\
7 & 37 & LI & Level VI & None & 0 & 0 & $<5$ & Conventional \\
8 & 37 & Total & Level VI & None & 0 & 0 & $<5$ & Conventional \\
\hline
\end{tabular}

${ }^{\mathrm{a} C}$ Cases 1 and 2 correspond to cases 1 and 2 in Table 2 .

Ex, significant extrathyroid extension evaluated on intraoperative findings; pEx, significant extrathyroid extension evaluated on pathological examination; $\mathrm{cN}$, clinical nodal status at the time of surgery; $\mathrm{pN}$, pathological nodal status; LIs, labeling indices; LI, lobectomy with isthmectomy (hemithyroidectomy).

size. This patient remained under active surveillance because her PMC was diminished in size again at the next examination one year later. The size of the tumor of case 4 was measured in the 3rd trimester, and it had not enlarged at that time. After delivery, an enlargement of $3 \mathrm{~mm}$ was observed, but the size of the PMC did not increase further in the 36 months after the delivery.

\section{Patients who underwent surgery after observation}

To date, seven patients, including two whose PMCs enlarged during their pregnancies, have undergone surgery after delivery (Table 3 ). Cases 1 and 2 in Table 3 are the same as cases 1 and 2 in Table 2, respectively. Cases 3 and 7 underwent surgery because of patient preference, although they displayed no signs of progression during pregnancy. Patient 4 was advised to undergo surgery because of the coexistence of Graves' disease. Patient 8 underwent surgery because of enlargement (from 65 to $123 \mathrm{~mm}$ in size) of an associated nodular goiter in the contralateral lobe. These patients (numbers 3, 4, 7, and 8) underwent surgery within one year after delivery.

At the beginning of her active surveillance, patient 5 had enlargement of level VI lymph nodes, which were thought to be reactive nodes due to chronic thyroiditis. Although the size and number of nodes did not change from before to after the pregnancy, the attending physician performed a FNAB for one of the nodes 20 months after delivery. Since it was diagnosed as metastasis, the patient underwent surgery. The $\mathrm{PMC}$ in patient 6 was stable during pregnancy, but a lymph node diagnosed as metastasis by FNAB at level IV newly appeared more than three years after delivery. Therefore, this patient also underwent surgery.

\section{Pathological findings of the eight patients who underwent surgery}

The histology of seven of the tumors, including one with size enlargement, was conventional papillary carcinoma (Table 3). Three patients, including one with size enlargement during pregnancy (case 1), were $\mathrm{pN}$-positive, whereas the other five were $\mathrm{pN}$-negative. None of the patients had significant extrathyroidal extension corresponding to pT4a in the UICC TNM classification, and the Ki-67 labeling indices (LIs) of all tumors were low at $<5 \%$, suggesting slow growth of these tumors. The histology of the remaining tumor was diagnosed as papillary carcinoma with multiple honeycomblike small cysts, as indicated above (14).

\section{Discussion}

Shindo et al. from Kuma Hospital reported that low-risk PMCs enlarged significantly more frequently during pregnancy compared with an age-adjusted control group (44.4\% vs. $11.1 \%$ ) (13). They encountered four patients with PMCs that showed an increase in size during pregnancy, and they added the cases of five additional pregnant patients with PMCs and compared the change in the size of these patients' PMCs with those of age- and sex-adjusted non-pregnant patients with PMCs. They specifically looked at the change in tumor size during gestation, as they suspected that the risk of tumor enlargement might increase during pregnancy. Since this is a clinically important issue, all of the patients with PMCs who had a pregnancy and delivery during their active surveillance at Kuma Hospital were herein reviewed.

Cases 1 and 2 in Table 2 in the present study had a significant tumor enlargement during pregnancy, which were appropriately described by Shindo et al. (13). Among the other cases judged as showing enlargement during pregnancy, one decreased in size after delivery to near the size observed before pregnancy, and the other was not considered to have an enlargement after the re-evaluation by the sonographer (H.O.). Since Shindo et al. collected a small number of patients, selection bias might have occurred. In addition, the aim of the study by Shindo et al. was mainly to investigate whether the size of PMCs changes during pregnancy (13). The aim of the present study, in contrast, was to determine whether pregnancy and delivery significantly affect the progression of low-risk PMCs.

In this study, in order to avoid selection bias, the cases of the patients with pregnancy and delivery were extracted from all female patients who underwent active surveillance between 1993 and 2013 at Kuma Hospital. Fifty such patients were identified with a total of 51 events. The PMCs of all 50 patients were measured before their pregnancies and after their deliveries as described in the Patients and Methods section. The analysis revealed two major findings. First, 5/51 PMCs ( $8 \%$ ) grew by $\geq \geq 3 \mathrm{~mm}$ during pregnancy, but only two 
(4\%) patients underwent surgical resection after delivery because the size of the remaining two PMCs was stable after delivery. Second, none of the cases showed novel lymph node metastasis. One patient (2\%) showed a decrease in tumor size after delivery. One case was diagnosed as having level VI lymph node metastasis proven by fine-needle aspiration cytology 20 months after delivery, but since the size and the number of enlarged nodes did not change and the patient had chronic thyroiditis, it remains unclear whether lymph node metastasis occurred during pregnancy. Another patient showed a novel appearance of level IV metastasis, but it occurred more than three years after delivery, and therefore seems unrelated to her pregnancy.

High serum levels of hCG during pregnancy might stimulate the growth of low-risk PMCs as suggested by Shindo et al., but in this study, this phenomenon was observed at a much lower incidence $(8 \%)$ than that reported by Shindo et al. (44.4\%) (13). It is speculated that after delivery, at which time the serum hCG levels again become undetectable, the growth of the PMCs stopped or even reversed in one-half of the cases who had some enlargement during pregnancy.

In summary, the present findings demonstrate that the incidence of growth of PMCs during pregnancy and delivery is low at $8 \%$ (4/50 cases), and that none of the patients developed lymph node metastasis during their pregnancies, although lymph node metastases developed 20 months after delivery in one patient. Therefore, despite the fact that longterm surveillance should be performed and further studies with larger series of pregnant patients are needed to draw more reliable conclusions, it can currently be concluded that PMC growth during pregnancy is not a common event, suggesting that a possible future pregnancy does not preclude patients with PMCs from undergoing active surveillance, although watchful observation during the pregnancy is recommended.

\section{Author Disclosure Statement}

No competing financial interests exist.

\section{References}

1. Ito Y, Miyauchi A 2007 A therapeutic strategy for incidentally detected papillary microcarcinoma of the thyroid. Nat Clin Pract Endocrin Metab 3:240-248.

2. Takebe K, Date M, Yamamoto Y 1994 Mass screening for thyroid cancer with ultrasonography [in Japanese]. KARKINOS 7:309-317.

3. Ito Y, Uruno R, Nakano K, Takamura Y, Miya A, Kobayashi K, Yokozawa T, Matsuzuka F, Kuma S, Kuma K, Miyauchi A 2003 An observation trial without surgical treatment in patients with papillary microcarcinoma of the thyroid. Thyroid 13:381-388.
4. Sugitani I, Koda K, Yamada K, Yamamoto N, Ikenaga M, Fujimoto Y 2010 Three distinctly different kinds of papillary thyroid microcarcinoma should be recognized: our treatment strategies and outcomes. World J Surg 34:1222-1231.

5. Ito Y, Miyauchi A, Inoue H, Fukushima M, Kihara M, Higashiyma T, Tomoda C, Takamura Y, Kobayashi K, Miya A 2010 An observation trial for papillary thyroid microcarcinoma in Japanese patients. World J Surg 34:28-35.

6. Ito Y Miyauchi A, Kihara M, Higashiyama T, Kobayashi $\mathrm{K}$, Miya A 2014 Patient age is significantly related to the progression of papillary microcarcinoma of the thyroid under observation. Thyroid 24:27-34.

7. Davies L, Welch HG 2006 Increasing incidence of thyroid cancer in the United States, 1973-2002. JAMA 595:21642167.

8. Davies L, Welch HG 2014 Current thyroid cancer trends in the United States. JAMA Otolaryngol Head Neck Surg 140:317-322.

9. Ahn HS, Kim HJ, Welch HG 2014 Korea's thyroid-cancer "epidemic"-screening and overdiagnosis. New Eng J Med 371:1765-1767.

10. Bellabio M, Poshychinda M, Ekins RP 1991 Pregnancyinduced change in thyroid function: role of human chorionic gonadotropin as putative regulator of maternal thyroid. J Clin Endocrinol Metab 73:824-831.

11. Gibellin B, Zamperini P, Proh M, Giogliano G 2011 Management and follow-up of thyroid cancer in pregnant women. Acta Otorhinolaryngol Ital 1:358-365.

12. Kimura M, Amino N, Tamaki H, Mitsuda N, Miyai K, Tanizawa O 1990 Physiologic thyroid activation in normal early pregnancy is induced by circulating hCG. Obstet Gynecol 75:775-778.

13. Shindo H, Amino N, Ito Y, Kihara M, Kobayashi K, Miya A, Hirokawa M, Miyauchi A 2014 Papillary thyroid microcarcinoma might progress during pregnancy. Thyroid 24:840-844.

14. Kobayashi K, Hirokawa M, Yabuta T, Fukushima M, Kihara M, Takamura Y, Ito Y, Miya A, Amino N, Miyauchi A 2013 Papillary thyroid carcinoma with honeycomb-like multiple small cysts: characteristic features of ultrasonography. European Thyroid J 2:270-274.

Address correspondence to: Akira Miyauchi, MD, PhD

Department of Surgery Kuma Hospital

8-2-35, Shimoyamate-dori

Chuo-ku

Kobe 650-0011

Japan

E-mail: miyauchi@kuma-h.or.jp 